\title{
Spectral methods plasticity modeling for fatigue damage estimation: experimental application - uniaxial case
}

\author{
H. Rognon ${ }^{1, a}$, T. Da Silva Botelho ${ }^{1}$, I. Tawfiq ${ }^{1}$ And M. Bennebach ${ }^{2}$ \\ 1 LISMMA (EA2336) Supméca, 3 rue Fernand Hainaut, 93407 Saint-Ouen Cedex, France \\ 2 CETIM, 52 avenue Félix Louat, BP 80067, 60304 Senlis Cedex, France
}

Received 18 September 2012, Accepted 17 January 2014

\begin{abstract}
In this paper, we present techniques for fatigue damage evaluation using spectral methods and a model taking into account confined elasto-plastic behavior. The model is associated with a local fatigue approach, covering the whole endurance domain (low cycle and high cycle fatigue). It uses Neuber's method and is valid for limited plasticity. To validate this modeling, we perform a correlation between spectral methods, modified spectral methods and experimental tests. Results presented here are focused on the uniaxial loading case.
\end{abstract}

Key words: Spectral methods / plasticity / random process / rainflow count / fatigue damage

\section{Introduction}

The phenomenon of fatigue has become a major design criterion in the engineering industry, requiring fatigue methods that better model real physical phenomena. Conventional fatigue methods are defined in time domain because their loading input data are usually a stress or strain time history. Algorithms such as Rainflow [1,2] extract stress cycles from the time history to obtain a stress distribution. To determine the lifetime generated by the stress distribution, we can use a fatigue law such as Basquin equation and a damage accumulation rule such as Palmgren-Miner law. Nevertheless, these methods require knowing the stress time history or the envelope of this time history.

In the case of random vibrations, the mechanical structure may have a dynamic response. It is difficult to know the stress time history and to make the classical fatigue analysis. In time domain, it is only calculated the damage associated with one realization of the random vibrations process. If one wants the damage caused by random processes (in this paper, only stationary ergodic random processes are considered), it is necessary to calculate damages on several different realizations of the same stochastic process. This approach is called Monte Carlo simulation. The main drawback of Monte Carlo simulations is the computation time [3].

\footnotetext{
${ }^{a}$ Corresponding author: herve.rognon@supmeca.fr
}

To overcome these problems it is suitable to work in the frequency domain. In addition, we model the random vibrations as a gaussian stochastic process, characterized by its PSD. Fatigue spectral methods are entirely defined in the frequency domain. The average damage is then calculated from the PSD of stress. The stress cycles distribution is not calculated but is estimated with a probability density function. This probability density depends on parameters of the PSD and the selected spectral method. Spectral methods have shown their reliability in fatigue life predictions, however, their theory requires several assumptions about the structure response (Fig. 1a) that limit their applications.

Our proposal extends the range of SM to low cycle fatigue, by modeling confined plastic behavior of materials and we use a fatigue law that take into account this plasticity. Figure 1b defines the framework in which we will expand the use of spectral methods. In the presence of non-linearity, the response of the structure is a stationary ergodic non-gaussian stochastic process. Although the assumption of zero mean is no longer true, we make the assumption that the response of the structure with a nonlinearity is zero mean because the non-linearity (plasticity) is confined.

To allow comparison between the proposed method and the methods of bibliography, we conducted vibration fatigue tests which will serve as a reference. Tests will be performed in the case of a nearly uniaxial response to be consistent with the definition of the methods studied. 


\section{Nomenclature}

\begin{tabular}{|ll|}
\hline$E[\bullet]$ & Mathematical expectation \\
$f$ & Frequency (Hz) \\
$G_{x x}(f)$ & Power spectral density of $x(t)$ \\
$R_{x x}(\tau)$ & Autocorrelation function of $x(t)$ \\
$\lambda_{m}$ & Spectral moment of order $m$ \\
$\nu_{0}$ & Number of zero-upcrossings \\
$\nu_{a}$ & Average number of maxima \\
$\gamma$ & Irregularity factor \\
$N$ & Number of cycles (lifetime) \\
$\sigma$ & Stress amplitude \\
$\sigma_{m}$ & Mean stress \\
$\varepsilon$ & Strain amplitude \\
$C, k$ & Material parameters of Basquin model \\
$\mathbf{E}$ & Young's modulus \\
$\varepsilon_{f}$ & Fatigue ductility coefficient \\
$\sigma_{f}$ & Fatigue strength coefficient \\
$b, c$ & Material parameters of Manson-Coffin-Basquin model \\
$h(P, V)$ & Joint probability density function of peak and valley \\
$P_{a}(\sigma)$ & Amplitude probability density function of $\sigma(t)$ \\
$d$ & Damage at a given amplitude $\sigma$ \\
$E[d]$ & Average damage in the interval $\sigma$ and $\sigma+$ d $\sigma$ \\
$E[D]$ & Average damage \\
$\mathrm{Tf}$ & Life time (seconds, hours or days) \\
$X(t)$ & Gaussian stochastic process \\
$Z(t)$ & Non-Gaussian stochastic process \\
$G(\bullet)$ & Direct transformation \\
$G$ & Inverse transformation \\
$M O Y \bullet$ & Average of stationary ergodic stochastic process $\bullet(t)$ \\
$S T D \bullet$ & Standard deviation of the stationary ergodic stochastic process $\bullet(t)$ \\
$S$ & Skewness \\
$K$ & Kurtosis \\
$h_{3}, h_{4}$ & Parameters of Hermite model \\
$k^{\prime}$ & Cyclic strength coefficient \\
$n^{\prime}$ & Cyclic strain hardening exponent \\
$\varepsilon_{e_{i}} \sigma_{e_{i}}$ & Strain and stress obtained by elastic behavior for class i \\
$\varepsilon_{r_{i}} \sigma_{r_{i}}$ & Strain and stress obtained by Neuber's model for class i \\
$H(\bullet \bullet$ & Cycles distribution with linear behavior \\
$H_{e p}(\bullet)$ & Cycles distribution with elasto-plastic behavior \\
&
\end{tabular}

\section{Acronyms}

\begin{tabular}{|ll|}
\hline PSD & Power Spectral Density \\
PDF & Probality Density Function \\
RMS & Root Mean Square \\
SM & Spectral Method \\
SMM & Spectral Method Modified \\
HM & Hermite Model + spectral method \\
\hline
\end{tabular}

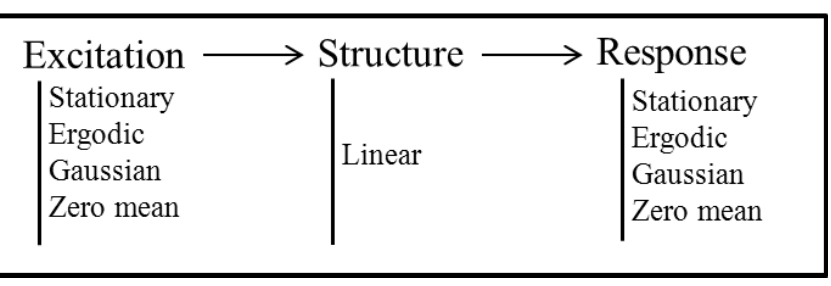

(a) Assumptions necessary for using spectral methods

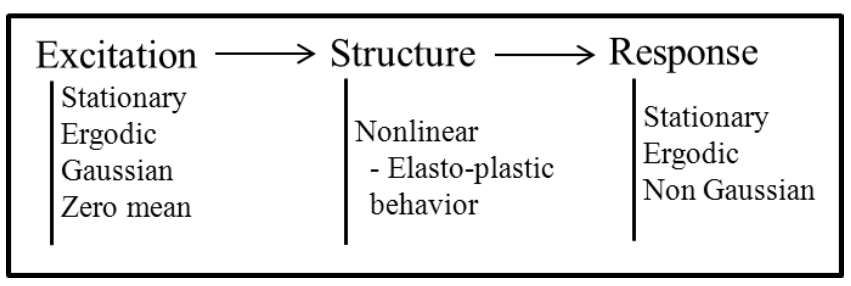

(b) Assumptions of our case study

Fig. 1. Definition of required assumptions in spectral methods and positionning of our framework. 


\section{General purpose}

\subsection{Properties of stochastic processes}

Power spectral density

In the case of a stationary Gaussian stochastic process, the PSD contains information defining this process [4]. Moreover, if the process is ergodic, the PSD can be calculated from the autocorrelation function of a realization of the random process $x(t)$ as follows, equation (1):

$$
G_{x x}(f)=\int_{-\infty}^{+\infty} R_{x x}(\tau) \mathrm{e}^{-i 2 \pi f \tau} \mathrm{d} \tau
$$

where $R(\tau)=E[x(t) x(t+\tau)]$. This equation (1) is known as the Wiener-Khintchine theorem.

\section{Spectral moments and signal parameters}

The spectral moments are important characteristics of a random process, they are calculated by equation (2).

$$
\lambda_{m}=\int_{-\infty}^{+\infty} G_{x x}(f)|f|^{m} \mathrm{~d} f
$$

They are related to the amount of information contained in a random process in the time domain. If for example $x(t)$ is a displacement and a random process with zero mean, $\lambda_{0}$ is the variance of the displacement, $\lambda_{2}$ is the variance of the velocity and $\lambda_{4}$ is the variance of the acceleration.

Spectral moments determine signal parameters of random processes. These results were demonstrated by Rice [5]. The number of zero-upcrossings is defined in equation (3).

$$
\nu_{0}=\sqrt{\frac{\lambda_{2}}{\lambda_{0}}}
$$

We define in equation (4) the number of maxima as:

$$
\nu_{a}=\sqrt{\frac{\lambda_{4}}{\lambda_{2}}}
$$

Another useful parameter is the irregularity factor. It represents the bandwidth of the PSD. It is calculated from the number of maxima and the number of zeroupcrossings, equation (5).

$$
\gamma=\frac{\nu_{0}}{\nu_{a}}=\sqrt{\frac{\lambda_{2}}{\lambda_{0} \lambda_{4}}}
$$

\subsection{Fatigue law}

Spectral methods commonly use the Basquin fatigue model [6]. This gives a power law relation between stress amplitude and life. This model leads to a linear StressLife curve in a log-log scale. It is defined in equation (6).

$$
N \sigma^{k}=C
$$

In the case of the proposed approach, the fatigue model used is the Basquin-Manson-Coffin's formulation [6]. This is a non linear model that determines total strain amplitude as a function of life. It is governed by equation (7):

$$
\varepsilon=\frac{\sigma_{f}^{\prime}}{\mathbf{E}}(2 N)^{b}+\varepsilon_{f}^{\prime}(2 N)^{c}
$$

\subsection{Spectral methods}

In a classical approach of damage, counting methods define each stress cycle extracted by a peak of level $\mathrm{P}$ and valley of level $\mathrm{V}$ or amplitude $\sigma$ and mean value $\sigma_{m}$. Each extracted cycle is a random event that has its own probability of occurrence. Consider $h(\mathrm{P}, \mathrm{V})$ the joint probability density of cycles extracted functions of peaks $\mathrm{P}$ and valley $\mathrm{V}$. By simple variable change, the joint distribution of cycles is obtained in terms of amplitude $\sigma$ and mean value $\sigma_{m}$, equation (8):

$$
P_{a, m}\left(\sigma, \sigma_{m}\right)=2 h\left(\sigma_{m}+\sigma, \sigma_{m}-\sigma\right)
$$

The amplitude probability density function (PDF) is then, equation (9):

$$
P_{a}(\sigma)=\int_{-\infty}^{+\infty} P_{a, m}\left(\sigma, \sigma_{m}\right) d \sigma_{m}
$$

Damage due to each stress amplitude $\sigma$, according to Basquin equation is equation (10):

$$
d=\frac{1}{N}=C^{-1} \sigma^{k}
$$

The expectation of damage contribution of cycles whose amplitude is between $\sigma$ and $\sigma+\mathrm{d} \sigma$, equation (11):

$$
E[d]=C^{-1} \nu_{a} \sigma^{k} P_{a}(\sigma) \mathrm{d} \sigma
$$

where $\nu_{a} P_{a}(\sigma) \mathrm{d} \sigma$ is the number of maxima per unit time in the interval $[\sigma ; \sigma+\mathrm{d} \sigma]$.

The damage expectation per unit time for the law of Palmgren-Miner is equation (12).

$$
E[D]=C^{-1} \nu_{a} \int_{0}^{+\infty} \sigma^{k} P_{a}(\sigma) \mathrm{d} \sigma
$$

$P_{a}(\sigma)$ is the amplitude probability density function and depends on the formulation made by each author. In our case, we use the probability density function (PDF) defined by Lalanne (Rice theory) [7] and Dirlik (Rainflow approach) [8]. 


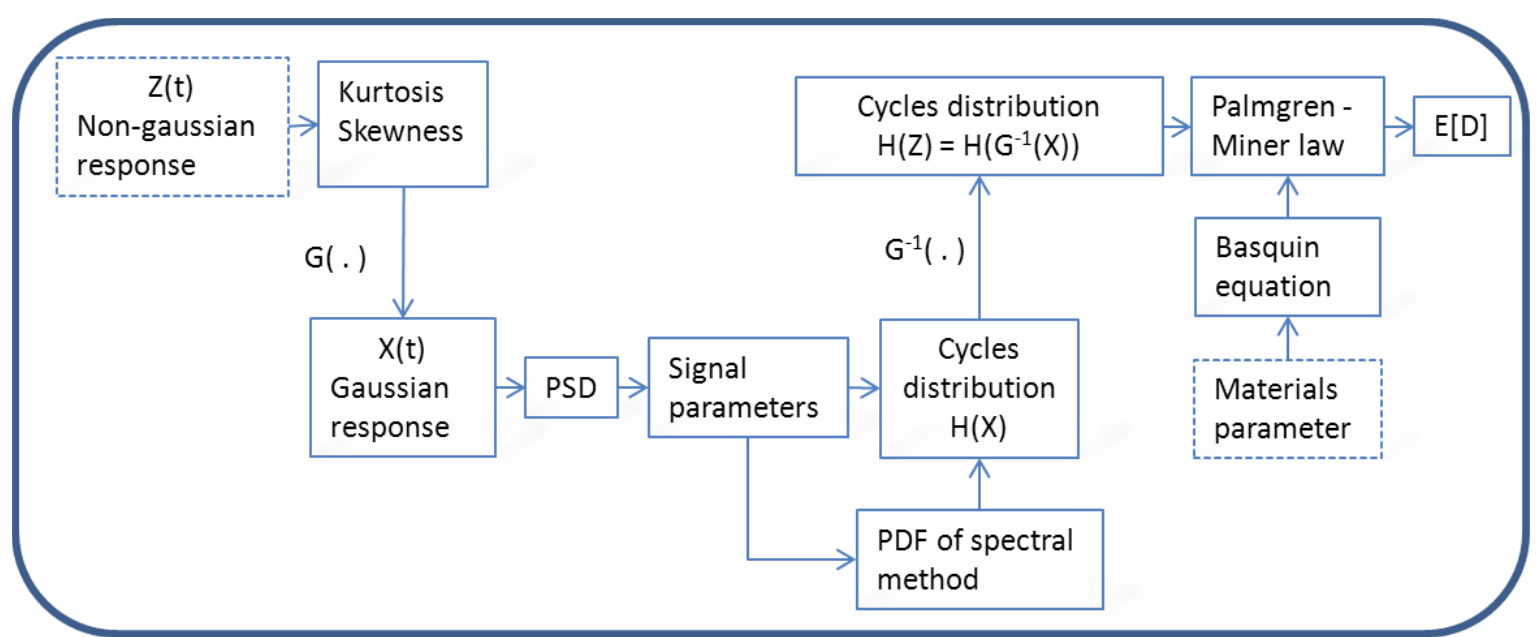

Fig. 2. Methodology diagram for calculating fatigue for non-gaussian process with a reversible transformation of the Hermite model type.

\section{Lalanne's method}

Lalanne [7] defines the amplitude probability density function from the work of Rice [5], given in equation (13).

$$
\begin{aligned}
P_{a}^{L a l}(\sigma)= & \sqrt{\frac{1-\gamma^{2}}{2 \pi \lambda_{0}}} \exp \left(\frac{-\sigma^{2}}{2 \lambda_{0}\left(1-\gamma^{2}\right)}\right) \\
& +\frac{\gamma \sigma}{2 \lambda_{0}} \exp \left(-\frac{\sigma^{2}}{2 \lambda_{0}}\right) \\
& \times\left[1+\operatorname{Erf}\left(\frac{\gamma \sigma}{\sqrt{2 \lambda_{0}\left(1-\gamma^{2}\right.}}\right)\right]
\end{aligned}
$$

where $\operatorname{Erf}(x)$ is the error function, defined by $\operatorname{Erf}(x)=$ $\frac{2}{\sqrt{\pi}} \int_{0}^{x} e^{-\alpha^{2}} \mathrm{~d} \alpha$. The average damage can be calculated from the equation (14).

$$
E\left[D^{\mathrm{Lal}}\right]=C^{-1} \nu_{a} \int_{0}^{+\infty} \sigma^{k} P_{a}^{L a l}(\sigma) \mathrm{d} \sigma
$$

\section{Dirlik's method}

In 1985, Dirlik [9] proposed to determine directly from the PSD the amplitude density probability based on the definition of a rainflow cycle. He determines the rainflow cycles probability density empirically, from a large number of Monte Carlo simulations with different forms of PSD and irregularity factors. The probability density by Dirlik is given in equation (15).

$$
\begin{aligned}
P_{a}^{\text {Dir }}(\sigma)= & \frac{1}{\sqrt{\lambda_{0}}}\left[\frac{D_{1}}{Q_{D}} \exp \left(-\frac{\varphi}{Q_{D}}\right)+\frac{D_{2} \varphi}{R^{2}} \exp \left(-\frac{\varphi^{2}}{2 R^{2}}\right)\right. \\
& \left.+D_{3} \varphi \exp \left(-\frac{\varphi^{2}}{2}\right)\right]
\end{aligned}
$$

where

$$
\begin{aligned}
\varphi & =\frac{\sigma}{\sqrt{\lambda_{0}}} ; \quad x_{m}=\frac{\lambda_{1}}{\lambda_{0}} \sqrt{\frac{\lambda_{2}}{\lambda_{4}}} ; \quad D_{1}=\frac{2\left(x_{m}-\gamma^{2}\right)}{1+\gamma^{2}} \\
R & =\frac{\gamma-x_{m}-D_{1}^{2}}{1-\gamma-D_{1}-D_{1}^{2}} ; \quad D_{2}=\frac{1-\gamma-D_{1}-D_{1}^{2}}{1-R} \\
D_{3} & =1-D_{1}-D_{2} ; \quad Q_{D}=\frac{1.25\left(\gamma-D_{3}-\left(D_{2} R\right)\right)}{D_{1}}
\end{aligned}
$$

Equation (17) indicates the average damage with the cycles probability density by Dirlik.

$$
E\left[D^{D i r}\right]=C^{-1} \nu_{a} \int_{0}^{+\infty} \sigma^{k} P_{a}^{D i r}(\sigma) \mathrm{d} \sigma
$$

\subsection{Hermite's model}

In the case if the structure response is a stationary ergodic non-Gaussian stochastic process, there are ways to perform the calculation of the fatigue damage. Hermite's model $[10,11]$ is a reversible transformation that can transform a non-Gaussian process in Gaussian process. Combined with spectral methods, this allows fatigue life calculation of a non-Gaussian stochastic process $[12,13]$. The global methodology is described in Figure 2. In our case, the non-Gaussian processes can be generated by a structure with a non-linear behavior. Here we use the Hermite model because it is easy to implement but there are other models such as Ochi and Ahn [14,15] and Power-law model [16].

Equation (18) represents the $G(\bullet)$ transformation [17] applied to our problem where $X_{0}$ is the reduced centered variable $\left(X_{0}=\frac{X-M O Y_{x}}{S T D_{x}}\right)$.

$X_{0}=\left[\sqrt{\xi^{2}(Z)+\eta_{3}}+\xi(Z)\right]^{\frac{1}{3}}-\left[\sqrt{\xi^{2}(Z)+\eta_{3}}-\xi(Z)\right]^{\frac{1}{3}}-\eta_{1}$ 


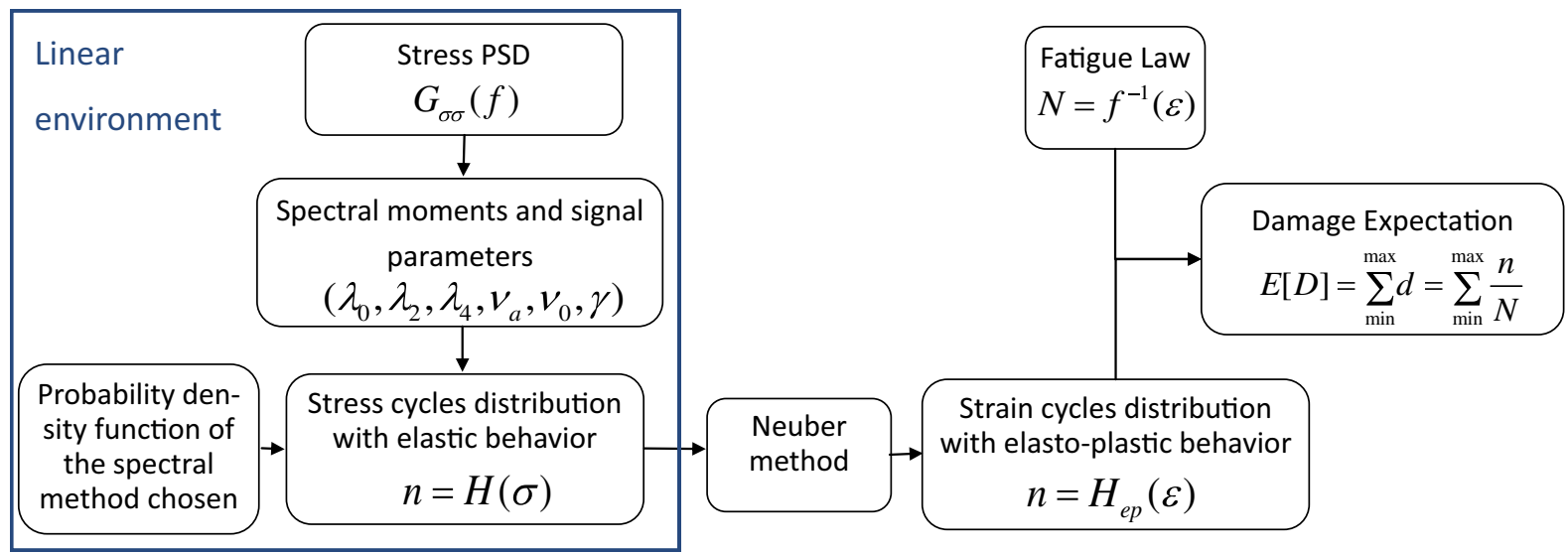

Fig. 3. Proposed fatigue process in the spectral methods.

where

$$
\begin{aligned}
\xi(Z) & =1.5 \eta_{2}\left(\eta_{1}+\frac{Z-M O Y_{z}}{S T D_{z}}\right)-\eta_{1}^{3}, \quad \eta_{1}=\frac{h_{3}}{3 h_{4}} \\
\eta_{2} & =\frac{1}{3 h_{4}}, \quad \eta_{3}=\left(\eta_{2}-1-\eta_{1}^{2}\right)^{3}, \quad h_{3}=\frac{S}{6} \\
h 4 & =\frac{(K-3)}{24}
\end{aligned}
$$

Equation (20) represents the inverse transformation of $\mathrm{G}$ $\left(G^{-1}\right)$ :

$Z=M O Y_{z}+S T D_{z}\left[X_{0}+h_{3}\left(\left(X_{0}^{2}-1\right)+h_{4}\left(\left(X_{0}^{3}-3 X_{0}\right)\right]\right.\right.$

\section{Modeling of plasticity in spectral methods}

\subsection{Modelization in spectral methods}

To take into account elasto-plastic behavior, the spectral methods need two essential elements to provide a good estimation of the damage. The first element is a fatigue law that takes into account the plastic behavior. The second element is a model giving the elasto-plastic local responses due to the loading. These two elements are taken into account by using the Basquin-Manson-Coffin model and the rule of Neuber $[18,19]$.

Manson-Coffin-Basquin's law can take into account the plastic behavior of the material during the accumulation of damage. Neuber's method defines a distribution that takes into account the elasto-plastic behavior from a linear calculation respecting the assumptions of spectral methods (Fig. 1). In spectral domain, it is difficult to access to the real strain time history, but for each strain level it is possible to obtain the cycles number corresponding, this is the distribution of cycles number ( $\mathrm{H}$ function). The $\mathrm{H}$ function is generally used for the discretized calculus.

Figure 4 shows how we apply for each stress increment the Neuber's method to obtain the real strain. To represent the present phenomenon, we use to apply the correction of Ramberg-Osgood equation and Neuber equation, see equation (21).

$$
\varepsilon_{r i}\left\{\begin{array}{c}
\varepsilon_{r i} \sigma_{r i}=\frac{\sigma_{e i} \sigma_{e i}}{\mathbf{E}} \longleftarrow \quad \text { Neuber } \\
\varepsilon_{r i}=\frac{\sigma_{r i}}{\mathbf{E}}+\left(\frac{\sigma_{r i}}{k^{\prime}}\right)^{1 / n^{\prime}} \longleftarrow \text { Ramberg-Osgood }
\end{array}\right.
$$

We must add the assumption that the total number of cycles from distribution remains unchanged regardless of the materials behavior [20]. The distribution is defined for a given time interval. In this time interval, the cycles number is given by the system frequency and not by the strain or stress amplitudes. We work only on the allocation of the cycles number on the space of possible real strain (Fig. 4). This means that the area under the curve remains the same before and after correction, only the cycles modification on the high strain modifies the damage [21]. This process is integrated in the process of spectral methods as shown in Figure 3. The assumptions of spectral methods are respected since it applies in the linear part of the process.

\section{Tests and results}

\subsection{Specimen description}

The specimen in Figure 5 is designed to meet the assumption of the method described previously (confined plasticity). It is made from quenched \& tempered $30 \mathrm{NiCrMo} 8$ steel, whose general properties are summarized in Table 1. The specimen consists of two parts (cylindrical portion and inertia block), crimped together by a shrink fitting process to allow transfer of forces. In Figure $5 \mathrm{c}$, we show in blue the clamping area of the specimen. The flat part prevents any rotation of the specimen during the test. The cylindrical portion of the clamping area allows for conditions well defined and reproducible on all specimens. Table 2 gives the experimental fatigue data for the laws of Basquin and Coffin-Manson-Basquin. The experimental value for the Basquin slope $k$ is 12.03 . To avoid dispersion due to choice of damage laws, we chose 
Table 1. Mechanical properties of 30NICRMO8.

\begin{tabular}{cccc}
\hline Young's modulus & Poisson's coefficient & Yield strength & Tensile strength \\
\hline $195 \mathrm{GPa}$ & 0.33 & $945 \mathrm{MPa}$ & $1135 \mathrm{MPa}$ \\
\hline
\end{tabular}

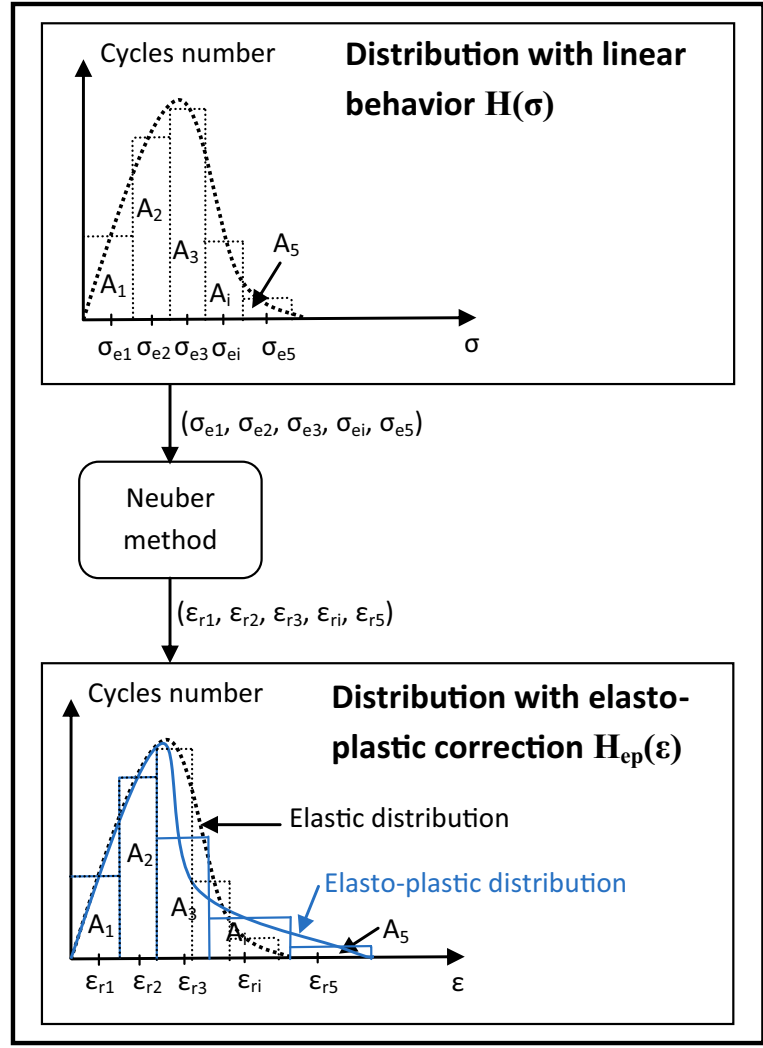

Fig. 4. Correction method for the distribution of cycles number in the spectral methods.

to adjust it to the slope of the elastic line from BasquinManson-Coffin $(1 / 0.085=11.764)$.

\subsection{Test bench description}

Each test is composed of three specimens (Fig. 6) to save time and set repetitive. The specimens are inserted in a clamping system. The excitation system is a electrodynamic vibrator which is controlled by several parameters (RMS value, level of the PSD). The tests are performed on the second eigenmode of the specimen in order to have an uniaxial loading. The PSD excitation is a PSD band $90 \mathrm{~Hz}-120 \mathrm{~Hz}$, centered around the eigenmode. Table 5 in first column presents the different levels of PSD selected for our tests. We have 9 specimens for each level, requiring 3 tests. Accelerometers are placed on the specimen in order to detect crack initiation.

The lifetime is defined as the time between the start of the test and the initiation of the crack. The crack initiation is detected by the variation of the frequency and amplitude of the eigenmode.
Table 2. Fatigue properties of $30 \mathrm{NiCrMo} 8$ steel.

\begin{tabular}{cccccc}
\hline \multicolumn{2}{c}{ S N parametrers } & \multicolumn{4}{c}{ E N parameters } \\
\hline$k$ & $C$ & $b$ & $c$ & $\varepsilon_{f}^{\prime}$ & $\sigma_{f}^{\prime}(\mathrm{MPa})$ \\
\hline 11.764 & $3.659 \times 10 \mathrm{e} 36$ & -0.085 & -0.752 & 1.741 & 1750.000 \\
\hline
\end{tabular}

\subsection{Numerical model and modal analysis}

To compare the experimental lifetimes for different numerical methods, we modeled the specimen using a finite element code (ABAQUS). This allows retrieving the stress frequency response function which is the input data for calculation methods in fatigue.

Numerical model was refined thanks to correlations with experimental data (density, Young modulus and damping). Table 3 shows the experimental eigenfrequencies from average of measurements performed on 10 test pieces (shock hammer and shaking table), we can see that the gap with the frequency of the numerical model is less than $1 \%$ in the case of our study. Figure 7 shows each modal shape with excitation along $\vec{Z}$, we note that the second eigenmode is orthogonal to the first and third eigenmodes.

A convergence study of the mesh was performed in order to ensure good estimation of stresses and strains in the critical area. We refined the mesh until the values of stress and strain does not vary between two refined meshings. We obtained that $41 \%$ of the degrees of freedom are located in the notch so that it represents only $5 \%$ of the total volume of the specimen.

We also performed a damping factor analysis. This parameter must be correctly quantified because it strongly affects the values of stress and strain. In our case, the damping is low, the slightest error for estimating it involves lifetimes completely wrong. To determine a damping value consistent across all tests, we calculate the damping of each test during the first two hours of testing where the specimen is healthy. We calculate the damping means on all tests. We chose the damping mean as damping value for our calculations. This value is 0.0016 or $0.16 \%$ damping.

\subsection{Results and observations}

The presented results are in the context of an excitation of the second eigenmode which generates a nearly uniaxial response. Table 4 summarizes the mean and standard deviation of the experimental life times by level of excitation. Means and standard deviations were calculated on a set of 36 specimens (9 specimens per level). Data are calculated in seconds and hours. The last column of the table indicates the ratio between mean and 


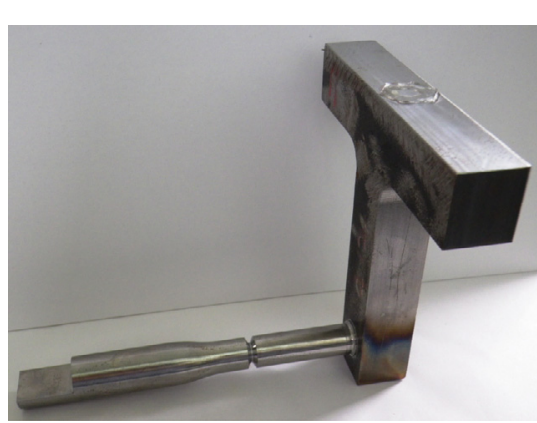

(a) Specimen global view

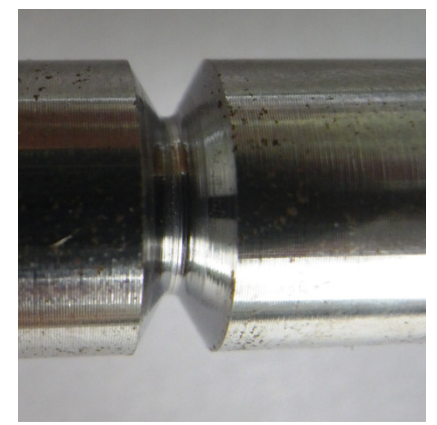

(b) View of the critical area of (c) CATIA model view with clamping the specimen

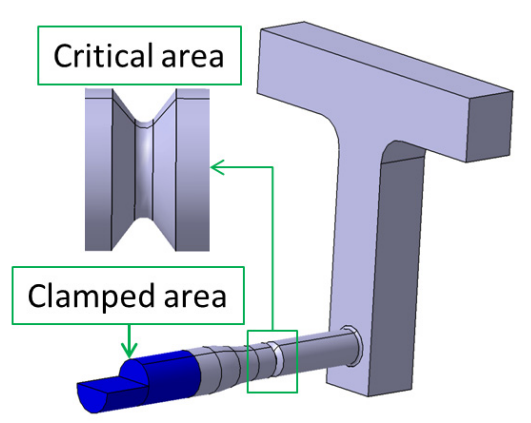

area

Fig. 5. Vibration fatigue test specimen.

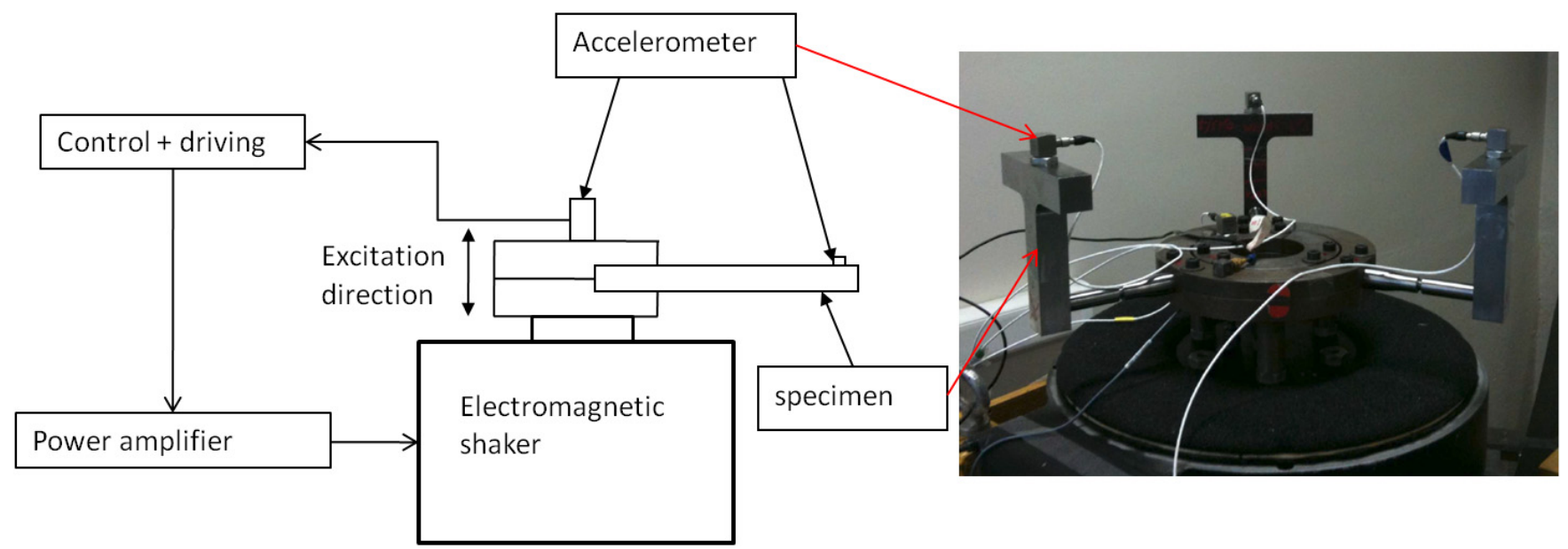

Fig. 6. Schema of test bench with the different elements that compose it and a picture of the test bench with specimens.

Table 3. Dynamic parameters (experimental and numerical data).

\begin{tabular}{cccccc}
\hline Eigenmode & $\begin{array}{c}\text { Experimental } \\
\text { eigenfrequency }(\mathrm{Hz})\end{array}$ & $\begin{array}{c}\text { Numerical } \\
\text { eigenfrequency }(\mathrm{Hz})\end{array}$ & Damping (\%) & Loading type & $\begin{array}{c}\text { Loading state } \\
\text { (in critical area) }\end{array}$ \\
\hline 1st & 94.5 & $94.2(-0.31 \%)$ & 0.11 & Bending and Torsion & multiaxial \\
2nd & 102.7 & $102.1(-0.58 \%)$ & 0.16 & Bending & uniaxial \\
3rd & 248.7 & $256.5(+3.14 \%)$ & 0.48 & Torsion and Bending & multiaxial \\
\hline
\end{tabular}

Table 4. Experimental average lifetime by excitation level well as the associated stantard deviation.

\begin{tabular}{|c|c|c|c|c|}
\hline Level of PSD acceleration $\left(\mathrm{g}^{2} / \mathrm{Hz}\right)$ & Lifetime (initiation) & Average & Standard deviation & Standard deviation/Average \\
\hline & Hours & 4.4 & 0.8 & \\
\hline 0.0040 & $\begin{array}{c}\text { Seconds } \\
\text { Hours }\end{array}$ & $\begin{array}{c}1.6+\mathrm{E} 04 \\
9.6\end{array}$ & $\begin{array}{c}2.8+\mathrm{E} 03 \\
1.2\end{array}$ & 0.175 \\
\hline 0.0034 & $\begin{array}{c}\text { Seconds } \\
\text { Heures }\end{array}$ & $\begin{array}{c}3.5 \mathrm{E}+04 \\
17.6\end{array}$ & $\begin{array}{c}4.2 \mathrm{E}+03 \\
2.2\end{array}$ & 0.120 \\
\hline 0.0030 & $\begin{array}{c}\text { Secondes } \\
\text { Heures }\end{array}$ & $\begin{array}{c}6.3 \mathrm{E}+04 \\
29.7\end{array}$ & $\begin{array}{c}7.8 \mathrm{E}+03 \\
5.0\end{array}$ & 0.124 \\
\hline 0.0026 & Secondes & $1.1 \mathrm{E}+05$ & $1.8 \mathrm{E}+04$ & 0.164 \\
\hline
\end{tabular}



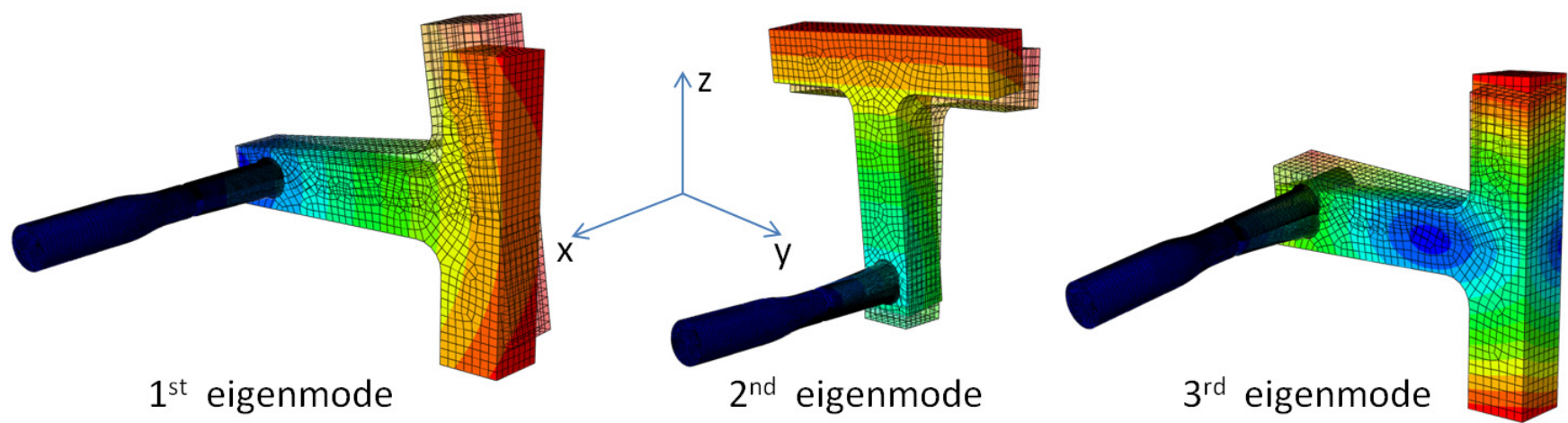

Fig. 7. Deformations of each eigenmode with in hard, the specimen at rest and in transparent, the specimen deformed.

Table 5. Comparaison between the experimental lifetimes and the lifetimes of the different methods, in parenthesis, the relative error from the experimental lifetime.

\begin{tabular}{|c|c|c|c|c|c|c|c|c|}
\hline \multirow{3}{*}{$\begin{array}{l}\text { Excitation } \\
\text { level } \\
\left(\mathrm{g}^{2} / \mathrm{Hz}\right)\end{array}$} & \multirow{3}{*}{$\begin{array}{l}\text { Experi- } \\
\text { mental } \\
\text { life }(s)\end{array}$} & \multicolumn{7}{|c|}{ Numerical data } \\
\hline & & \multirow{2}{*}{$\begin{array}{l}\text { RMS } \\
(\mathrm{MPa})\end{array}$} & \multicolumn{2}{|l|}{ SMM (s) } & \multicolumn{2}{|l|}{ SM (s) } & \multicolumn{2}{|c|}{ Hermite (HM) (s) } \\
\hline & & & Dirlik & Lalanne & Dirlik & Lalanne & Dirlik & Lalanne \\
\hline 0.0040 & $1.6 \mathrm{E}+$ & 229 & $1.2 \mathrm{E}+04(-25 \%)$ & 4 & $1.6 \mathrm{E}+02(-99.1 \%)$ & & $3.1 \mathrm{E}+03(-80.6 \%)$ & $\overline{2.91}$ \\
\hline 0.0034 & 3. & 211 & $3.4 \mathrm{E}+04(-2.9 \%)$ & $3.2 \mathrm{E}+04$ & $1.6 \mathrm{E}+03(-95.9 \%)$ & $1.5 \mathrm{E}$ & $1.4 \mathrm{E}+04(-60.0 \%)$ & $3.5 \mathrm{E}+04$ \\
\hline 0.0030 & $6.3 \mathrm{E}+$ & 197 & $5.7 \mathrm{E}+04(-9.5 \%)$ & $5.4 \mathrm{E}+04$ & $9.2 \mathrm{E}+03(-85.3 \%)$ & $8.7 \mathrm{E}$ & $3.8 \mathrm{E}+04(-39.6 \%)$ & $6.3 \mathrm{E}+04$ \\
\hline 0.0026 & $1.1 \mathrm{E}+05$ & 175 & $1.1 \mathrm{E}+05(+3.6 \%)$ & $1.0 \mathrm{E}+05$ & $7.6 \mathrm{E}+04(-30.9 \%)$ & $7.2 \mathrm{E}+04$ & $9.5 \mathrm{E}+05(-13.6 \%)$ & $1.1 \mathrm{E}+05$ \\
\hline
\end{tabular}

standard deviation. In our case, the ratio is quite low regardless of the level of excitation. It appears that there is no relationship between this ratio and the level of excitation. This gives a good level of confidence in the experimental results.

In Table 5, we compare experimental and numerical lifetimes for Lalanne and Dirlik models. In the second column, we reported the RMS value obtained by calculating the PSD stress. This value is the same regardless of the method selected because this is an input data for fatigue calculation. Third column gives the experimental lifetime average per excitation level. The lifetime average per level is obtained from nine specimens of three tests. The other columns are the lifetimes obtained from the numerical methods. The average lifetimes are calculated from the probability density function (PDF) of Lalanne and Dirlik. In case of Dirlik PDF, in parentheses, the values of relative errors are compared to experimental lifetimes. The relative error is defined in equation (22).

$$
\begin{aligned}
& \begin{array}{l}
\text { Relative error }(\%)= \\
\text { calculated lifetime }- \text { experimental lifetime }
\end{array} \\
& \text { experimental lifetime }
\end{aligned}
$$

For the proposed model (SMM), the relative errors compared to experimental lifetimes are low; it shows a good accuracy of the method. In contrast for classical spectral methods (SM), the maximum relative error is $99.1 \%$, it is obtained between the experimental lifetime and the lifetime with classical spectral method at the level 1 . The result is consistent because the lower the calculated lifetime, the more elastoplastic the material behavior is. Since the spectral methods ignore the elasto-plastic behavior, this implies skewed results. Conversely, in the case of level 4, the loads are small and the material works globally with a elastic behavior. In reference [22], for the steel $30 \mathrm{NiCrMo}$, a RMS value of less than $180 \mathrm{MPa}$ involves elastic behavior during the fatigue test. This explains good correlation between classical spectral methods predictions and experimental results.

Table 5 results are illustrated in Figure 8. As Xaxis there are the lifetimes obtained with the different numerical methods. As Y-axis, there are the lifetimes obtained experimentally. A point on the graph forms the combination of a life of a numerical method associated with the corresponding experimental life. A point on the median line means a simulation fitting perfectly experimental data. This allows to see that the main influence is due to material nonlinearity; the probability density function selected (Lalanne or Dirlik) has little effect. This highlights that it is the material nonlinearity that is in default in classical spectral methods.

We also observe that the Hermite model associated with spectral methods tends to give better results at any level than classical spectral methods. Nevertheless, this model diverges when the nonlinearity increases and moves away from the experimental results. The proposed model gives a good estimate of the lifetime of the specimen at any level. This shows the influence of the elasto-plastic behavior on the fatigue life.

\section{Conclusion and perspectives}

The proposed method allows a better estimation of the lifetime by including the influence of the plastic behavior of the material on the fatigue phenomenon. To consolidate 


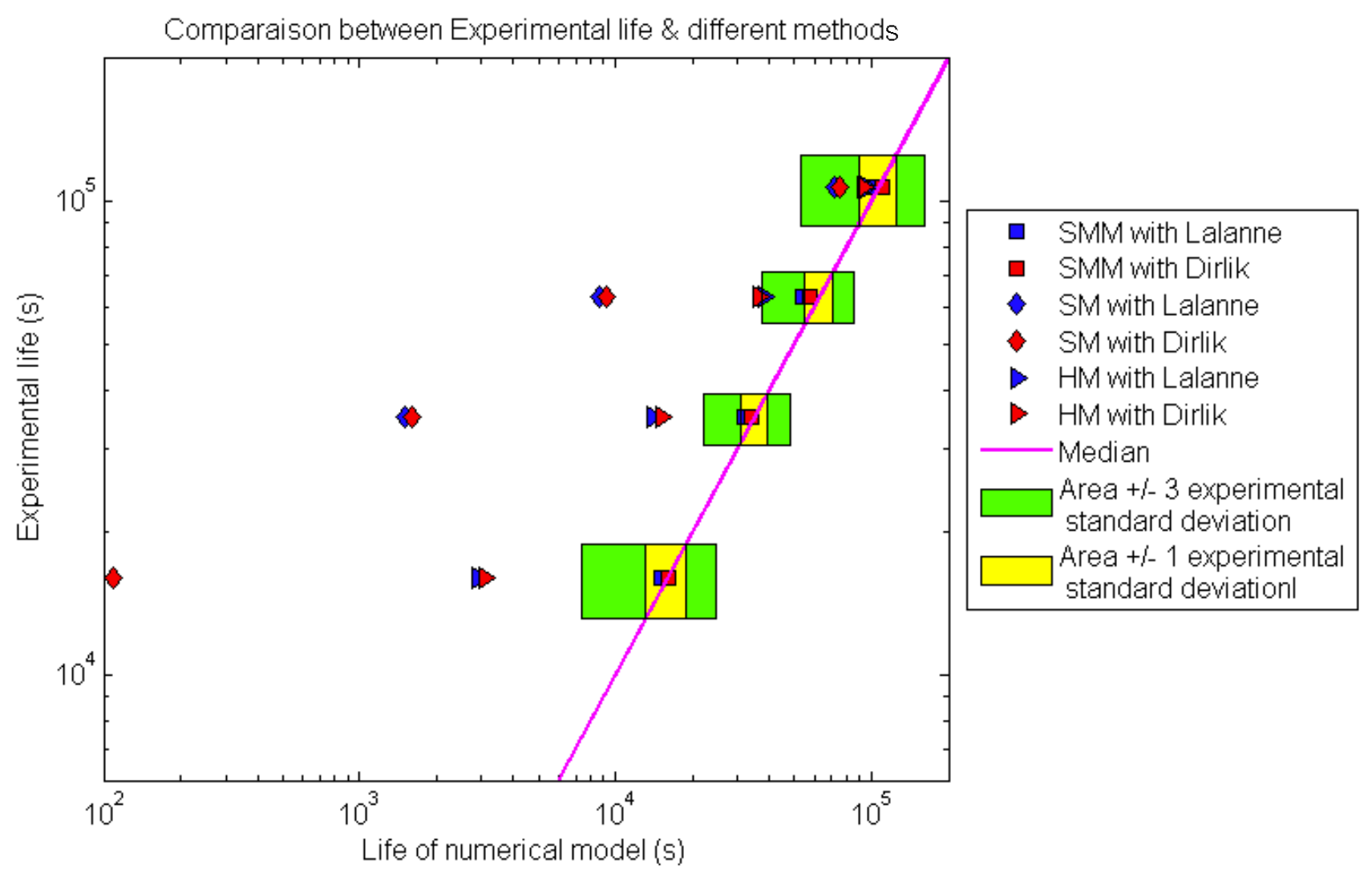

Fig. 8. Synthesis of different experimental and numerical liftimes for the specimen excited on the second eigenmode.

the results obtained, other tests will be perfomed for different levels and different types of solicitation.

We found that the Hermite model gives good results but tends to diverge when the nonlinearity increased. There may be two reasons for this. The first is that the method does not model directly the nonlinearity. The second is that the fatigue law used is a $\mathrm{S}-\mathrm{N}$ law that does not take into account the plasticity.

We have shown that if the classical spectral methods give incorrect results, this was due to the non-respect of assumptions, in our case, the linearity of the material behavior.

For the following, we have several axes of improvement:

- We will study the impact of experimental and numerical scatterings on the different lifetimes results. This involves both the variability of experimental lifetimes and of numerical lifetimes. We also study the impact of the damping scatterings on the calculated lifetimes.

- We will study and model the inclusion of non reversed strains (non-zero mean of the process studied). And, we will focus on the modeling of hysteresis phenomenon of material in the fatigue calculation.

Acknowledgements. We are grateful to the CETIM Foundation for funding this work on the fatigue behavior under vibration environment. We also thank the SUPMECA Instrumentation Service for his help during the tests.

\section{References}

[1] AFNOR, Fatigue sous sollicitations d'amplitude variable. Méthode rainflow de comptage, 1993

[2] S. Downing, D. Socie, Simple rainflow counting algorithms, Int. J. Fatigue 4 (1982) 31-40

[3] X. Pitoiset, Méthodes spectrales pour l'analyse en fatigue des structures métalliques sous chargements aléatoires multiaxiaux. Ph.D. thesis, Université libre de Bruxelles, 2001

[4] A. Preumont, Vibrations aléatoires et analyse spectrale. Presses Polytechniques Romandes, 1990

[5] S. Rice, Mathematical analysis of random noise. Selected papers on noise and stochastic processes, 1954

[6] J. Lemaitre, J. Chaboche, Mécanique des matériaux solides. Dunod, 1996

[7] C. Lalanne, Mech. Vib. Shock Analysis: Fatigue Damage, Lavoisier, 2009, Vol. 4

[8] D. Benasciutti, R. Tovo, Comparison of spectral methods for fatigue analysis of broad-band gaussian random processes, Probabilistic Engineering Mechanics 21 (2006) $287-299$

[9] T. Dirlik, Application of computers in fatigue analysis. Ph.D. thesis, University of Warwick, 1985 
[10] S. Winterstein, Non-Normal Responses And Fatigue Damage, J. Eng. Mech. ASCE 111 (1985) 1291-1295

[11] S. Winterstein, T. Ude, T. Marthinsen, Volterra Models Of Ocean Structures - Extreme And Fatigue Reliability, J. Eng. Mech. ASCE 120 (1994) 1369-1385

[12] D. Benasciutti, R. Tovo, Cycle distribution and fatigue damage assessment in broad-band non-Gaussian random processes, Prob. Eng. Mech. 20 (2005) 115-127

[13] D. Benasciutti, R. Tovo, Fatigue life assessment in nonGaussian random loadings, Int. J. Fatigue 28 (2006) 733746

[14] M. Ochi, Probability distributions of peaks and troughs of non-Gaussian random processes, Prob. Eng. Mech. 13 (1998) 291-298

[15] M. Ochi, K. Ahn, Probability distribution applicable to non-gaussian random processes, Prob. Eng. Mech. 9 (1994) 255-264

[16] S. Sarkani, D. Kihl, J. Beach, Fatigue of welded-joints under narrow-band non-gaussian loadings, Prob. Eng. Mech. 9 (1994) 179-190
[17] S. Winterstein, Nonlinear vibration models for extremes and fatigue, J. Eng. Mech. ASCE 114 (1988) 1772-1790

[18] H. Neuber, Theory of stress concentration for shearstrained prismatic bodies with arbitrary nonlinear stressstrain law., J. Appl. Mech. 28 (1961) 544-551

[19] G. Glinka, Calculation of inelastic notch-tip strain stress histories under cyclic loading, Eng. Fract. Mech. 22 (1985) 839-854

[20] H. Rognon, Comportement en fatigue sous environnement vibratoire: Prise en compte de la plasticité au sein des méthodes spectrales. Ph.D. thesis, Ecole Centrale Paris, 2013

[21] H. Rognon et al., Modeling of plasticity in spectral methods for fatigue damage estimation of narrowband random vibrations, in IDETC/CIE (ASME, ed.), (Washington DC, USA), 2011

[22] H. Rognon et al., Modeling of plasticity in spectral methods for fatigue damage estimation of random vibrations., in Fatigue Design (CETIM, ed.), (Senlis, French), 2011 